\title{
Resolving the spatial relationship between intracellular components by dual color super resolution optical fluctuations imaging (SOFI)
}

\author{
Maria Elena Gallina ${ }^{1,2}$, Jianmin Xu', Thomas Dertinger ${ }^{1,3}$, Adva Aizer ${ }^{4}$, Yaron Shav-Tal $^{4}$ and Shimon Weiss ${ }^{*}$
}

\begin{abstract}
Background: Multi-color super-resolution (SR) imaging microscopy techniques can resolve ultrastructural relationships between- and provide co-localization information of- different proteins inside the cell or even within organelles at a higher resolution than afforded by conventional diffraction-limited imaging. While still very challenging, important SR colocalization results have been reported in recent years using STED, PALM and STORM techniques.

Results: In this work, we demonstrate dual-color Super Resolution Optical Fluctuations Imaging (SOFI) using a standard far-field fluorescence microscope and different color blinking quantum dots. We define the spatial relationship between hDcp1a, a processing body (P-body, PB) protein, and the tubulin cytoskeletal network. Our finding could open up new perspectives on the role of the cytoskeleton in PB formation and assembly. Further insights into PB internal organization are also reported and discussed.
\end{abstract}

Conclusions: Our results demonstrate the suitability and facile use of multi-color SOFI for the investigation of intracellular ultrastructures.

Keywords: Dual color, Superresolution, Fluorescence microscopy, SOFI, P-bodies

\section{Background}

Despite the availability of microscopy methods that offer nanoscale resolution (like electron and scanning probe microscopies), far-field fluorescence microscopy (FFFM) is nevertheless the most commonly used imaging tool in biology. This primacy is due to FFFM's unique advantages, including the ability to work with live specimens, excellent bio-specificity and sensitivity, minimal perturbation, direct display and visualization of the specimen, and versatility and simplicity of usage. FFFM is therefore the preferred tool for investigating spatial organization of cell components. However, Abbe's intrinsic diffraction barrier limits the resolving power of the optical microscope to about half the excitation (and/or emission) wavelength, masking important information on morphology and co-localization

\footnotetext{
*Correspondence: sweiss@chem.ucla.edu

'Department of Chemistry and Biochemistry, Department of Physiology, and the California NanoSystem Institute (CNSI), University of California Los

Angeles, Los Angeles, CA 90095-1569, USA

Full list of author information is available at the end of the article
}

of cellular components. Recently, several ground-breaking super-resolution (SR) imaging techniques (Bates et al. 2007; Betzig et al. 2006; Hess et al. 2006; Hell 2003; Hell and Wichmann 1994; Gustafsson 2000; Heintzmann et al. 2002) have been developed, and with their aid, previously unresolved biological questions have found new answers (Bates et al. 2007; Donnert et al. 2007; Shroff et al. 2007; Huang et al. 2008). The dissemination and wide-adaptation of SR over the last decade has been phenomenal, pointing to the revolutionary potential of these methods. Nonetheless, first generation commercial (and even-home-built) SR microscopes are expensive, and are not yet simplified to the 'push-button' level that affords the facile use by the non-expert. Additionally, these methods often require long acquisition (and therefore exposure) times and relatively high intensity excitation/depletion/photo switching lasers that limit the applicability to photo-resistant samples. Stochastic techniques such as PALM (Betzig et al. 2006) and STORM (Rust et al. 2006; Bates et al. 2007) are restricted to the use of photo-switchable emitters. A 
continuous effort has been expended in recent years to further simplify SR techniques and make them more affordable. In particular, methods that rely on conventional (and already deployed) microscopy platforms and standard fluorophores have been pursued (Burnette et al. 2011; Simonson et al. 2011; Dertinger et al. 2009; Dertinger et al. 2010a). In this context, Super-resolution Optical Fluctuations Imaging (SOFI) (Dertinger et al. 2009; Dertinger et al. 2010a; Dertinger et al. 2010b; Dertinger et al. 2012b; Dertinger et al. 2012a; Geissbuehler et al. 2011; Geissbuehler et al. 2012) offers the attractive possibility of performing SR imaging with a standard FFFM and blinking fluorescent probes such as quantum dots (QDs) (Dertinger et al. 2009; Dertinger et al. 2010a; Dertinger et al. 2012b), the fluorescent proteins Dronpa and rsTagRFP (Dedecker et al. 2012), and even non-fluorescent blinking nanoplasmonic probes (in press). SOFI is based on high-order spatiotemporal statistical analysis of stochastic blinking of independent emitters or scatterers (Dertinger et al. 2009) recorded in a sequence of frames. Multiple order SOFI analysis, combined with re-weighting of the Optical Transfer Function (OTF) (or with deconvolution (Dertinger et al. 2010a)), increases the resolution over the diffraction limit by a factor of $n, n$ being the correlation (cumulant) order. In addition, spatiotemporal cross-cumulants calculation leads to an increase in the numbers of pixels that constitute the SOFI (SR) image (Dertinger et al. 2010a). In this work, we show a new procedure for performing two-color SOFI (2cSOFI) on fixed cells by using different color light emitting QDs. In particular, we demonstrate that 2cSOFI can effectively resolve the spatial relationship between the microtubule cytoskeleton and hDcpla, a constitutive processing body (P-body, PB) protein. PBs (Liu et al. 2005; Sen and Blau 2005) are recently discovered protein-RNA aggregates, implicated in degradation, storage and silencing of mRNAs. PBs appear to be spatially confined along the microtubule network (Aizer et al. 2008), which in turn seems to regulate their formation and assembly (de Heredia and Jansen 2004; Shav-Tal and Singer 2005). Therefore, knowledge of the spatial correspondence between these two intracellular structures is of particular interest. In addition, PBs are ideally suited, due to their small dimensions (a few hundreds of nanometers), to be studied by SR imaging.

\section{Methods}

2cSOFI imaging was performed on fixed, wild-type HeLa cells and on U2OS osteosarcoma cells, stably transfected with a GFP-hDcpla fusion protein and selected for a moderate expression level (Aizer et al. 2008). Instead of performing regular immunofluorescence with a primary and secondary antibody, the labeling was performed with primary antibodies directly conjugated to QDs. This affords higher density labeling, which is important for SR imaging. The average diameter of QDs conjugates was measured to be $c a .22 \mathrm{~nm}$ (considerably smaller than the resolution afforded by SOFI). PBs were labeled with 625-nm emitting QDs conjugated to anti-hDcpla primary antibody (rabbit) (Abcam, USA); the tubulin network was labeled with 800-nm emitting QDs conjugated to anti-alpha-tubulin primary antibody (rabbit) (Sigma-Aldrich, USA). Control experiments for antibody selectivity were performed to validate the conjugates (see Additional file 1: Figure S1), in particular the co-localization of anti-hDcpla QD conjugates with GFP-hDcpla was used to confirm the labeling of cytoplasmic PBs (see Figure 1a-c). Since QD blinking obeys a characteristic on/off power law distribution, fluorescence fluctuations could be observed on all time scales (Kuno et al. 2001). This property is particularly useful since good matching between blinking rate and camera frame rate is easily achieved. Together with their intrinsic high brightness and excellent photostability, QDs are well suited for SOFI imaging. The two color data sets were acquired sequentially by changing filter-sets while maintaining the focal plane unchanged. Movies of 2000 frames were collected for each color. Chromatic aberrations and misalignment between channels were minimized, and measured to be well below the achieved SOFI resolution (see Microscope Set Up and Data Analysis); the absence of stage drifting during movie acquisition was verified by the use of fiducial markers (data not shown).

\section{Bioconjugation}

Amine-derivatized, PEG-coated 800 and 625-nm QDs were purchased from Invitrogen (Grand Island, USA). Monoclonal anti-alpha-tubulin primary antibody (mouse) was purchased from Sigma-Aldrich (USA), monoclonal anti-hDcp1a primary antibody (rabbit) was purchased from Abcam (USA). Bioconjugation was performed at room temperature by amine-thiol cross-linking: sulfosuccinimidyl 4-(N-maleimidomethyl) cyclohexane-1carboxylate (Sulfo-SMCC) (Thermo Scientific, USA) was used to couple the thiol groups of biomolecules to the amino-terminated quantum dots. Thiolation of antibodies was obtained by reducing disulfide crosslinks of cysteine units in proteins with dithiothreitol (Thermo Scientific, USA). $4 \mu \mathrm{L}$ of $10 \mathrm{mM}$ sulfo-SMCC solution were added to a $4 \mu \mathrm{M}$ QDs solution, the mixture was incubated at room temperature for one hour. $2 \mu \mathrm{L}$ of $1 \mathrm{M}$ dithiothreitol were added to $1 \mathrm{mg} / \mathrm{ml}$ primary antibodies and kept for half an hour. After both components underwent purification by desalting columns, the conjugation reaction was allowed to proceed for one hour prior to quenching, obtained by adding 2-mercaptoethanol 


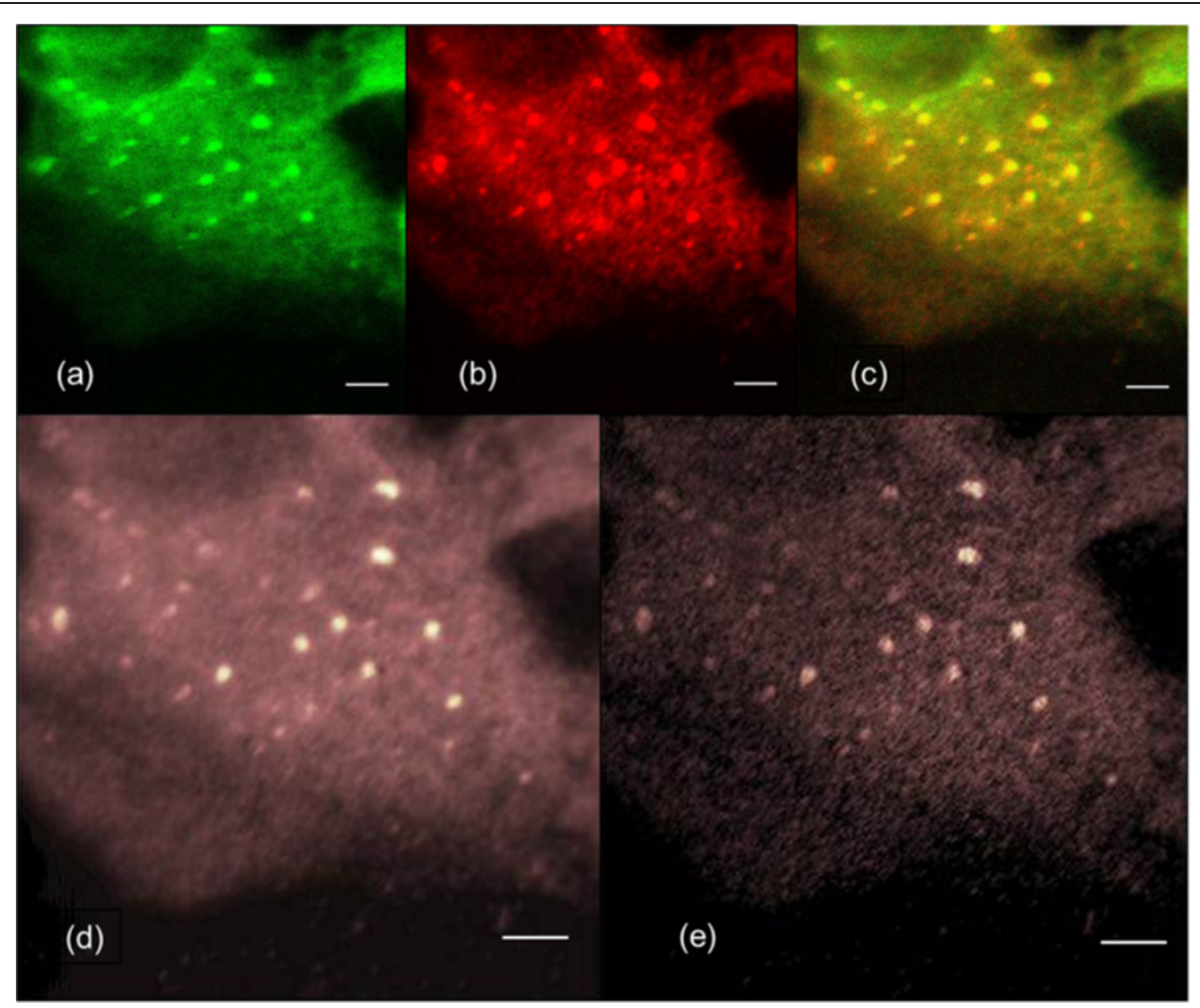

Figure 1 FFFM images ((a), (b) and (c)) of a U2OS cell treated with puromycin: (a) exogenous GFP-hDcp1a protein emission (green); (b) 625-nm QD anti-hDcp1a emission (red); and (c) co-localization of GFP and 625-QD. Co-localization of exogenous GFP-hDcp1a and endogenous hDcpla marked by 625-QD confirms the selectivity of the QD conjugate. Images do not reveal only the presence of PBs, distinguishable by their typical punctate appearance, but also a wide distribution of hDcpla proteins all over the cytoplasm, noticeable observing either GFP (a) or QD (b) emission. Indeed, Dcpla is located in both cytosol and PBs. (d) another FFFM of 625-nm QD anti-hDcpla, and (e) correspoding SOFI image. Scale bar=5.0 $\mu \mathrm{m}$.

(Invitrogen, USA) to the reaction mixture. After 30 minutes the solution was concentrated via ultra-filtration and purified by separation media column. The final concentration of bioconjugated QDs was $1 \mu \mathrm{M}$. Further details on the bioconjugation can be found on line: (http:// tools.invitrogen.com/content/sfs/manuals/mp19010.pdf).

\section{Cell culture and fixation}

Human cervical cancer cell line HeLa was ordered from American Type Culture Collection (ATCC, USA), the human osteosarcoma stable cell line U2OS expressing GFP-h-Dcp1a fusion protein was generated as described elsewhere (Aizer et al. 2008). HeLa and U2OS cells were cultured at $37^{\circ} \mathrm{C}, 5 \% \mathrm{CO}_{2}$ in high glucose DMEM medium (Invitrogen, USA), supplemented with 10\% Fetal Bovine Serum (FBS) and 1\% Pen Strep (5000 units/mL penicillin G, $50 \mu \mathrm{g} / \mathrm{mL}$ streptomycin sulfate in $0.85 \% \mathrm{NaCl}$ ). Cells were pre-cultured prior to experiments in glass-bottom Petri dishes until 60\% confluence was reached. The fixation was done incubating cells with cold fixative buffer (2 mM EGTA in 70\% v/v methanol, 20\% v/v acetone, $10 \% \mathrm{v} / \mathrm{v}$ water mixture) for $25 \mathrm{~min}$ at $-20^{\circ} \mathrm{C}$, after cells were rinsed with ice-cold PBS buffer. Cells were finally washed with TBS buffer. Stress conditions were induced on U2OS and HeLa cells immediately prior to the fixation, incubating cells for 1 hour at $37^{\circ} \mathrm{C}, 5 \% \mathrm{CO}_{2}$ with $100 \mathrm{ug} / \mathrm{mL}$ Puromycin (InvivoGen, France) dissolved in culture medium.

\section{Immunofluorescence labeling}

Labeling of cytosolic proteins was obtained by incubating primary antibody QDs conjugates dissolved in blocking buffer (2\% BSA, 0.05\% Tween-20 in TBS buffer) with methanol/acetone fixed cells for half an hour at $4^{\circ} \mathrm{C}$, following the protocol reported by Richard L. Ornberg and H. Liu (Weckwerth 2007). Cells were then washed with borate buffer and preserved in TBS buffer for microscopy measurements.

\section{Microscope setup and data analysis}

2000 frames movies were taken on a conventional LEDbased (Aura light engine, Lumencor Inc., USA) widefield microscope (Nikon Eclipse Ti, Nikon Inc., USA) with a $150 \times$ magnification obtained using a $100 \times$ objective (NA 1.49 oil) coupled with a $1.5 \times$ camera coupling lens. An EMCCD camera (Andor Ikon 897, Andor Technology, 
UK) was used to detect the signal, with exposure times of $30 \mathrm{~ms}$. Different color data sets were acquired sequentially by changing filter-sets: GFP [excitation (470/75), dichroic (505), emission (525/50)]; 625-nm QDs [excitation (535/ 50), dichroic (585), emission (620/40)]; 800-nm QDs [excitation (460-480 nm), dichroic (505), emission (700LP)]. All filters were purchased from Chroma Technology (USA). Multi-channel imaging of single color labeled cells indicated minimal crosstalk between the channels (below the detection limit of the EMCCD camera). Systematic errors in image registration between channels (due to consecutive alternation of the filter cube and chromatic aberrations) were corrected using a custom written Matlab (The MathWorks, Inc., USA) registration routine.

The registration routine is based on the alignment of signals collected from individual fluorescence microspheres (Invitrogen, USA) that emit in all channels and are evenly distributed over the field of view. In a first step, a registration matrix is originated by a projective transformation, and used to maximize the spatial correlation between different channels. In the next step, centers of individual PSFs are localized in all channels (using a 2D-Gaussian fit), and a second projective transformation is chosen to minimize the distances between corresponding PSF centers in the different channels (see Supporting Information for details). Additional file 1: Figure S5 gives a quantitative evaluation of the alignment accuracy, by showing a histogram of distances between centers of corresponding PFSs. Most of these shifts $(>95 \%)$ are smaller than 0.3 pixel $(32 \mathrm{~nm})$, well below 2nd order SOFI resolution. The reproducibility of this analysis was tested for 100 images per channel (i.e. consecutive and repetitive filter cube switching). The resulting transformation matrix was used for subsequent measurements. Movies were analyzed by a custom written Matlab (The MathWorks, Inc., USA) code that is described elsewhere (Dertinger et al. 2009; Dertinger et al. 2010a). Co-localization analysis of two color images was done using an ImageJ Plugin (Nakamura et al. 2007).
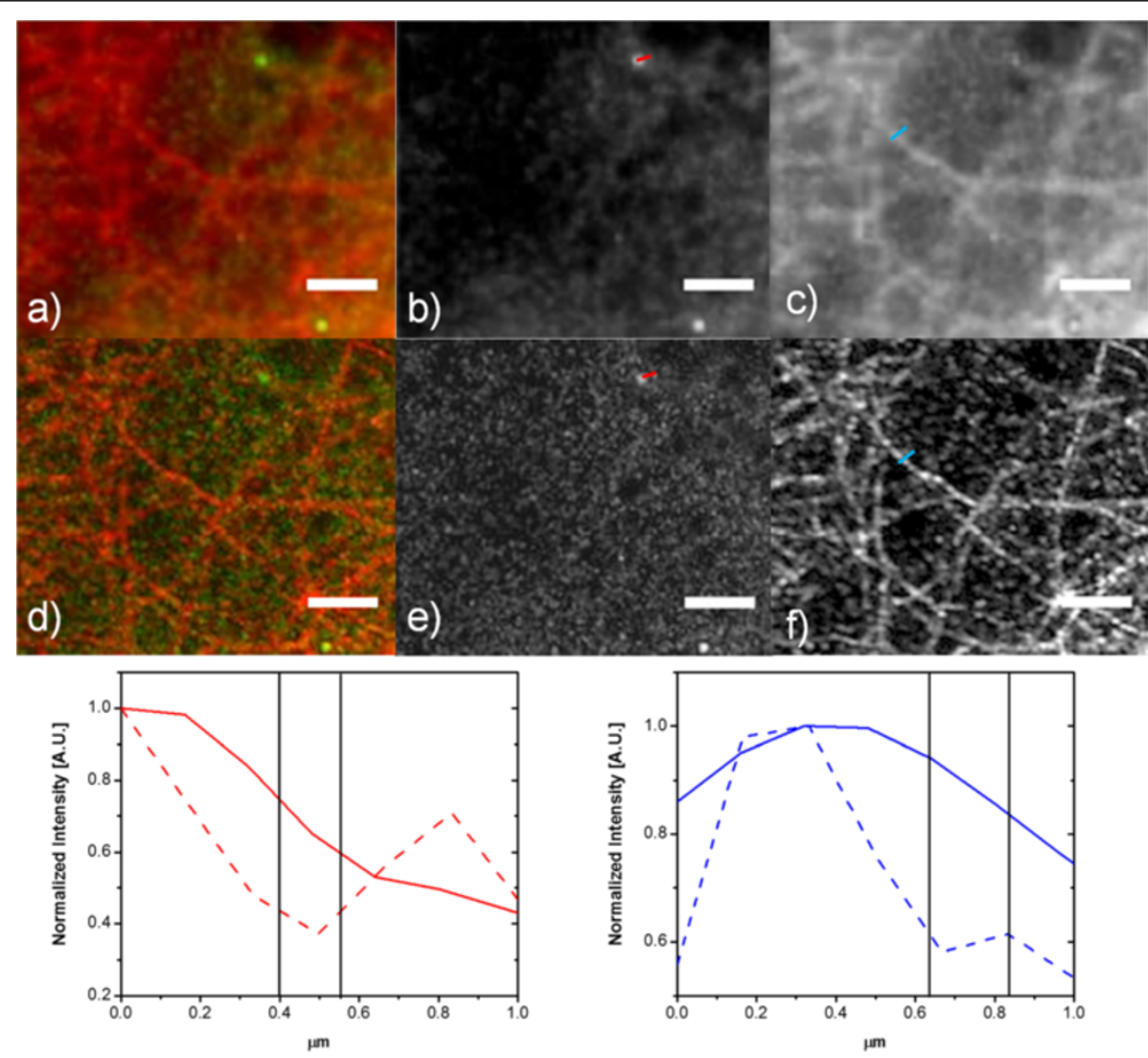

Figure 2 Co-localization between hDcp1a ((b) and (e)) and tubulin ((c) and (f)) in a HeLa cell, presented as a FFFM (a) and a SOFI overlay (d). Tubulin and Dcp1a were labeled with $800 \mathrm{~nm}$ and $625 \mathrm{~nm}$ QDs and are false-colored in red and green in (a) and (d), respectively. Lower panels: Cross-sections along the lines as indicated in the upper images. On the left: intensity profile of the region indicated by the red line in the FFFM (b) (red solid line) and the SOFI image (e) (red dashed line) of hDcpla labeled by 625-nm QDs. On the right: intensity profile of the region indicated by the blue line in the FFFM (c) (blue solid line) and the SOFI image (f) (blue dashed line) of alpha-tubulin labeled by $800-n m$ QDs. Scale bar: $(-)=5.0 \mu \mathrm{m}$. 


\section{Results and discussion}

Figure 2 illustrates representative $\alpha$-tubulin and hDcp1a conventional FFFM (Figure 2a) and SOFI (Figure 2d) co-localization data in a HeLa cell. The SOFI image (Figure 2d) exhibits not only appreciable increase in resolution and contrast, but also elimination of background noise and scattered light. Indeed, the removal of uncorrelated intensity values intrinsic to the SOFI analysis leads to an improvement of the signal to noise ratio. The SOFI-processed hDcp1a image (Figure 2e), as compared to the FFFM image (Figure 2b), unravels uniformly distributed sub-diffraction limited spots in the cytosol.

$\mathrm{PB}$ research in mammalian cells has focused on the large and easily detectable PBs (Figure 3g), rather than on these smaller structures that tend to blend in with the background (when imaged using FFFM). SOFI processing however shows that these actually represent individual or small aggregates of hDcpla monomers. In contrast, two larger aggregates, probably fully assembled PBs, can be observed in the upper-and lower-right parts of the figure (see FFFM and SOFI Figures $2 \mathrm{~b}$ and 2e, respectively). In order to quantify the resolution gain obtained by 2 cSOFI, we first calculated the theoretical resolution in conventional images $\left(R_{C}\right)$ using the Rayleigh criterion:

$$
R_{c}=\frac{0.61 \lambda}{N A}
$$

where NA is the numerical aperture of the objective and $\lambda$ is the emission wavelength. We found $R_{c}=330$ and 260 $\mathrm{nm}$ for the 800-nm and 625-nm light emitting QDs, respectively. Then we measured, for both channels, the distance between the cross-sections of features that were unresolved in the conventional fluorescence image, but were resolved by SOFI (lower panels in Figure 2). We found that the resolution of SOFI images $\left(R_{S}\right)$ is 200 for 800-nm and $155 \mathrm{~nm}$ for 625-nm light emitting QDs. The experimental resolution enhancement is somewhat lower than the two-fold increase expected for second-order cumulant analysis. This slight discrepancy is due to experimental limitations such as pixilation and noise which are not taken into account in $R_{c}$.

Figure 3 shows a typical FFFM (c) and SOFI (f) tubulin-hDcp1a co-localization in a U2OS cell. The hDcp1a aggregate in the upper-right part of the image is identified as a mature $\mathrm{PB}$ (verified by co-localization with exogenous GFP-hDcpla, data not shown). Interestingly, the morphology of the PB in the SOFI image is of a doughnut-shape (Figures 3e, $\mathrm{f}$ and i). Its external diameter is $c a .700 \mathrm{~nm}$, however no internal structure can be distinguished in the conventional fluorescence

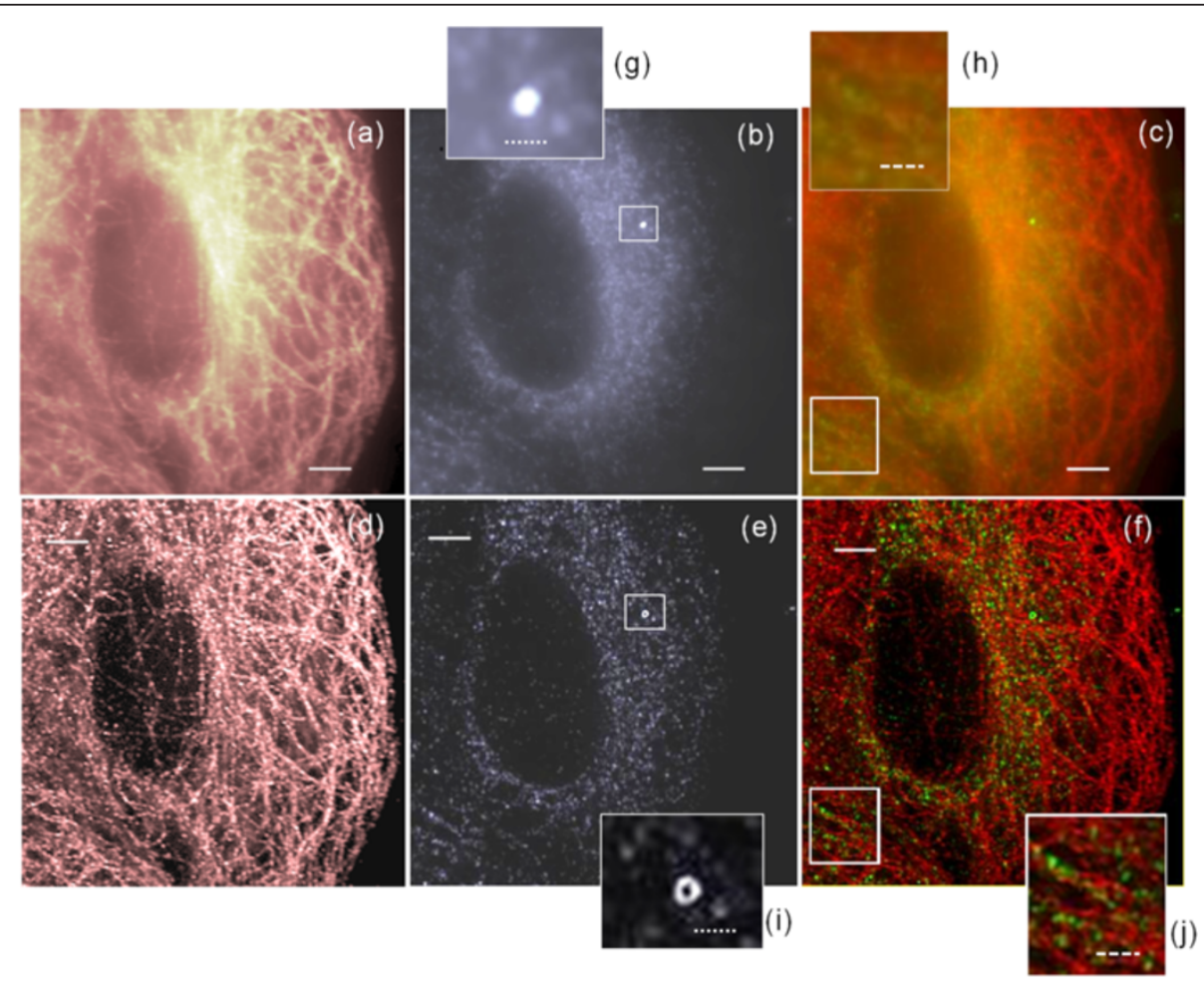

Figure 3 Co-localization between tubulin ((a) and (d)) and hDcp1a ((b) and (e)) in a U2OS cell, presented as a FFFM overlay (c) and as a SOFI overlay (f). The labeling sheme is analogous to Figure 2. Magnified views of the boxed regions in (b), (c), (e) and (f) are shown in (g), (h), (i), and (j), respectively. Scale bars: $(-)=5.0 \mu \mathrm{m} ;(---)=2.7 \mu \mathrm{m} ;(\ldots)=1.2 \mu \mathrm{m}$. 
image (Figure 3g). A simple explanation for the presence of the central dark spot in the SOFI image of the PB is pixel saturation at the PB's centre. However, we took measures to verify that no saturation of the fluorescent signal occurred in the conventional images; alternative explanations have been investigated and are further discussed below. A comparison between the zoomed-in FFFM overlay (Figure 3h) and SOFI overlay (Figure 3j) demonstrates the ability of SOFI to distinguish and colocalize the two cellular structures under investigation. In particular, Figure $3 j$ suggests the occurrence of spatial correlation between the microtubule network and the distribution of $\mathrm{hDcp} 1 \mathrm{a}$ proteins in the cytosol, which appears to be preferentially located along tubulin cytoskeleton tracks. The same correlation pattern was also found in other regions of the cytosol (Figure 3f). Figure 4 shows another representative FFFM and SOFI tubulin-hDcp1a co-localization data in a U2OS cell (same labeling scheme as in Figure 2). Here too a single PB could be observed (blue box in Figure 4b and zoom-in 4d). The PB colocalizes with a tubulin filament, and many such colocalizations were observed, suggesting possible anchoring of PBs to the tubulin cytoskeleton. This is in agreement with previous findings that $\mathrm{PB}$ dynamics is dependent on- and confined to- microtubules (Aizer et al. 2008). Such anchoring could in turn suggest a role of the latter in $\mathrm{PB}$ regulation. The PB diameter, estimated from Figure 4, is around $300 \mathrm{~nm}$, consistent with electron microscopy results obtained for immunogold-labled PBs (Yang 2004), but a doughnut shape was not observed in this case. Figure $4 \mathrm{~d}$ also shows the presence of individual or small aggregates of $\mathrm{hDcpla}$ monomers in close proximity to the $\mathrm{PB}$. This arrangement might indicate a potential dynamic exchange of monomers between the mature $\mathrm{PB}$ and the surrounding hDcpla monomers (or small aggregates), which are also spatially correlated with the tubulin filaments. The zoom-ins in Figures $4 \mathrm{c}$ and $4 \mathrm{e}$ also indicate spatial correspondence between the two proteins, while other areas of the cytosol (upper-right region of Figure 4b) lack such correlation, and exhibit variations in local concentrations, possibly due to uneven morphology of the overall cytosolic area at a single focal plane. The occurrence of spatial correlation was verified for different cells belonging to the same cell line, and for wild-type HeLa cells (Additional file 1: Figure S6). We quantified the colocalization of hDcpla and the tubulin cytoskeleton by calculating Pearson's correlation coefficient (r) in conventional and SOFI images of U2OS and HeLa cells. In
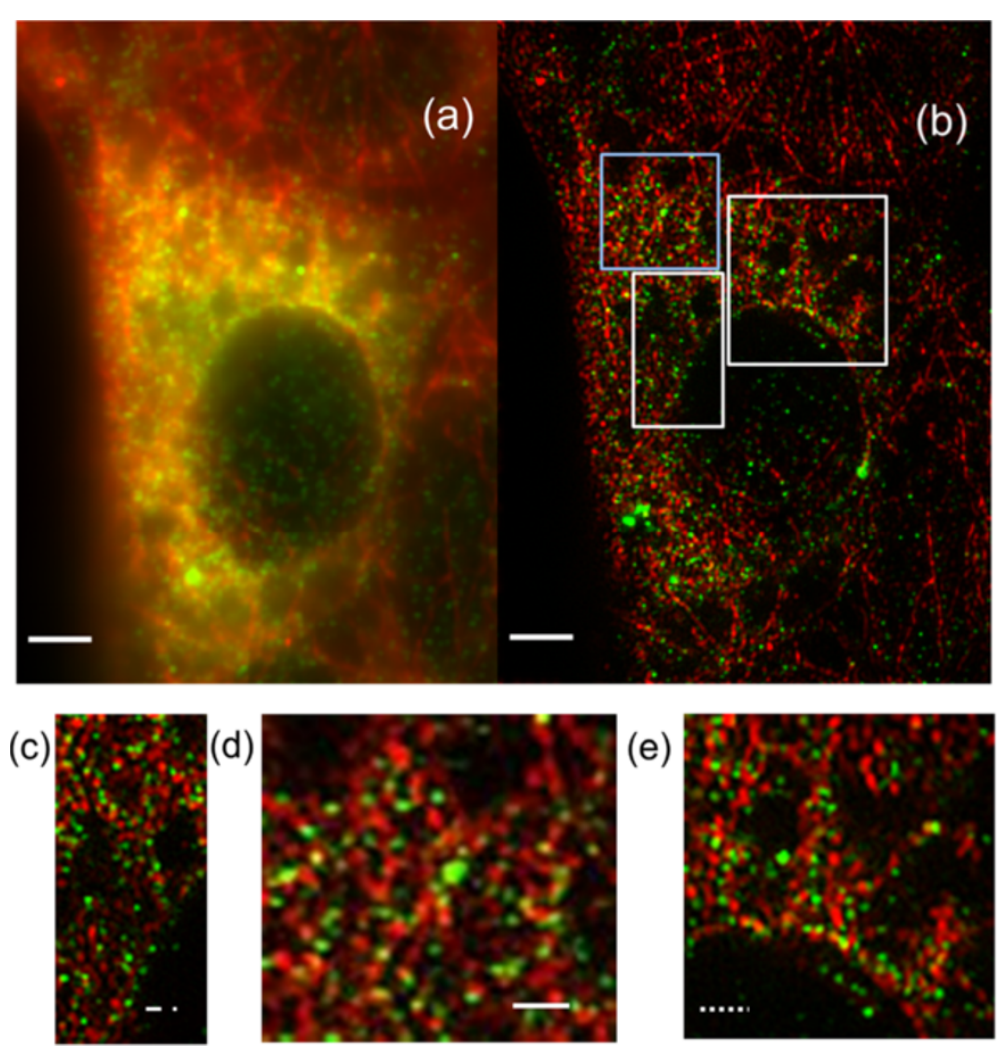

Figure 4 Co-localization between tubulin and GFP-hDcp1a in a U2OS cell, presented as a FFFM overlay (a) and as a SOFI overlay (b) and labeled the same way as in Figure 2. Boxed regions in (b) correspond to zoomed-in views (c), (d) and (e). Scale bars: $(-)=5.0 \mu \mathrm{m}$; $(-\cdot-)=1.6 \mu \mathrm{m} ;(---)=1.4 \mu \mathrm{m} ;(\ldots)=2.0 \mu \mathrm{m}$. 
statistics, the Pearson correlation coefficient $r$ is a measure of the linear dependence between two variables, giving a value that ranges between +1 and -1 . This parameter is widely used for statistical analysis, pattern recognition and image processing (Rodgers and Nicewander 1995, James 1988); possible applications comprise, for example, the comparison of two different images for image registration or object recognition purposes. For two gray scale images (denoted here in red and green images), the Pearson correlation coefficient $\mathrm{r}$ is described by the following equation:

$$
r=\frac{\sum_{i}\left(R_{i}-\bar{R}\right)\left(G_{i}-\bar{G}\right)}{\sqrt{\sum_{i}\left(R_{i}-\bar{R}\right)^{2}} \sqrt{\sum_{i}\left(G_{i}-\bar{G}\right)^{2}}}
$$

where $\mathrm{Ri}$ and $\mathrm{Gi}$ are the intensity of the ith pixel of the red and the green images, and $\bar{R}$ and $\bar{G}$ are the respective mean intensity values. $r$ equals +1 when the two images are identical, 0 when they are totally uncorrelated, and -1 when they are absolutely anti-correlated (i.e. one is the negative of the other). In our case, we found that the average Pearson coefficient is $\langle\mathrm{r}\rangle=0.87$ for conventional images and $\langle\mathrm{r}\rangle=0.60$ for their SOFI counterparts $(\mathrm{SD}=0.2, \mathrm{n}=7$ ). Even if, consistently with previous studies, SOFI detail enhancement results in a decrease in the Pearson correlation coefficient (from 0.87 to 0.60$),\langle\mathrm{r}>=0.60$ still represents a positive spatial correlation between the two proteins of interest. Additional quantification of this structural correspondence can be obtained using Manders coefficients M1 and M2 (Manders et al. 1993), which define the fraction of overlapping pixels belonging to two different color channels. They are defined as:

$$
M 1=\frac{\sum_{t} R_{i, \text { coloc }}}{\sum_{i} R_{i}} ; M 2=\frac{\sum_{t} G_{i, \text { coloc }}}{\sum_{i} G_{i}}
$$

where $R_{i, \text { coloc }}=R_{i}$ if $G_{i}>q$, and $G_{i, \text { coloc }}=G_{i}$ if $R_{i}>s$. M1 is the sum of the intensities of red pixels that have a green component divided by the total sum of red intensities, M2 is the analogous quantity calculated for green pixels; $\mathrm{q}$ and $\mathrm{s}$ are the threshold values set for the green and the red channel, respectively. M1 and M2 equal zero in absence of co-localization, +1 in case of perfect correspondence. We found that, for the conventional two color images reported in Figure 3, M1 $=0.36$ and $\mathrm{M} 2=0.66$, while for the corresponding 2cSOFI image we obtained M1 $=0.52$ and M2 $=0.79$. Analogously, the conventional two color image of Figure 4 and its SOFI counterpart gave, respectively, $\mathrm{M} 1=0.51, \mathrm{M} 2=0.57$ and M1 $=0.61, \mathrm{M} 2=0.69$. Here, the enhancement of resolution and of signal to noise ratio of SOFI data resulted in an increase of the parameters describing the spatial correlation. Indeed Manders coefficients are more sensitive to the background noise than the Pearson correlation coefficient and benefit more from image quality improvements. Also in this case, the obtained Manders coefficients support the presence of a positive spatial correlation between tubulin and hDcpla. This relationship might suggest the anchoring or interactions of $\mathrm{hDcp} 1 \mathrm{a}$ monomers/small aggregates with the cytoskeleton and could indicate a role for the tubular cytoskeleton in the regulation of $\mathrm{PB}$ formation and assembly (Shav-Tal and Singer 2005; de Heredia and Jansen 2004).

Based on immunogold electron microscopy experiments, PBs are generally described as non-membrane enclosed fibril aggregates of spheroidal shape (Yang 2004). On the contrary, SOFI imaging shows an unexpected doughnut-like appearance for several, but not all, detected PBs. In particular, 58\% of SOFI-processed images showed a ring-like structure with a central dip above the background noise level. These structures were not specific to the U2OS cell line (as observed, for example, in Figure 3i), but they were also observed in HeLa cells (Additional file 1: Figure S7). We further investigated $\mathrm{PB}$ ultra-structure by treating U2OS cells with puromicyn, an antibiotic known to increase the number and the size of PBs (Eulalio et al. 2007). The SOFI image (Figure 1e) indeed shows an increase in size and number, and partial loss of the circular shape for most PBs in the field-of-view. Interestingly, doughnutlike PBs were never observed in puromycin treated cells. Inconsistencies in the observed PB morphologies could be due to their different maturation stage. We confirmed that the doughnut-shape is not an artifact of the Fourier reweighting algorithm (see Additional file 1: Figure S7), but cannot rule-out artifacts due to experimental limitations such as insufficient resolution, incomplete labeling due to steric hindrance, and SOFI algorithmic issues.

On the other hand, the doughnut-shape morphology is consistent with a two-dimensional projection of a spherical shell organization of $\mathrm{hDcpla}$ around the $\mathrm{PB}$ core, a feature that is common to several other cellular compartments as, for example, sub-cellular protein vesicles (Bates et al. 2007) and promyelocytic leukemia nuclear bodies (PML-NBs) (Lang et al. 2010). Moreover, it is strongly corroborated by recent Fluorescence Recovery After Photobleaching (FRAP) experiments (Kedersha et al. 2005; Leung et al. 2006; Aizer et al. 2008). While in this study we cannot determine whether the doughnut shape of the PBs is of real structural significance, it should be noted that a bi-compartment model of the PB structure demarking a core region and an outer peripheral region has been demonstrated by electron microscopy (Weil et al. 2012). Moreover, several studies 
have shown that RNAs and factors can localize differentially to a peripheral domain distinguishable from a $\mathrm{PB}$ core domain (Weil et al. 2012, Pillai et al. 2005, Carbonaro et al. 2011). This compartmentalization might serve for the functional separation between a core area of degradational activity and a surrounding area of recruitment and storage and deserves further investigations by higher resolution techniques.

\section{Conclusions}

We demonstrated that dual color SOFI is suitable for SR morphology and SR co-localization studies of cellular components. The observed co-localization patterns between PBs and the microtubule network are in agreement with previous studies (Aizer et al. 2008). Furthermore, SOFI imaging revealed that cytosolic hDcpla monomers (or small aggregates) are preferentially located along tubulin filaments. This finding suggests that the role of the microtubule cytoskeleton is not limited to only anchoring $\mathrm{PBs}$, but possibly to also provide molecular tracks for monomer trafficking, delivery and exchange (Shav-Tal and Singer 2005; de Heredia and Jansen 2004; Aizer and ShavTal 2008).

\section{Additional file}

Additional file 1: Dual color super resolution optical fluctuations imaging (SOFI).

\section{Abbreviations}

2c: Two color; EMCCD: Electron-multiplying charge-coupled device; FFFM: Far-field fluorescence microscopy; FRAP: Fluorescence recovery after photobleaching; GFP: Green fluorescence protein; NA: Numerical aperture; OTF: Optical transfer function; PALM: Photo-activated localization microscopy; PB or P-body: Processing body; QD: Quantum dot; $R_{c}$ : Resolution in conventional FFFM images; $R_{s}$ : Resolution in SOFI images; SR: Superresolution; STED: Stimulated emission depletion; STORM: Stochastic optical reconstruction microscopy; SOFI: Super resolution optical fluctuations imaging; Sulfo-SMCC: Sulfo-succinimidyl 4-(N-maleimidomethyl) cyclohexane-1-carboxylate.

\section{Competing interests}

TD and SW are shareholder of SOFast GmbH Berlin, a company aiming for the commercial advancement and exploration of the SOFI technology. All other Authors declare that they do not have competing interests.

\section{Authors' contributions}

MEG designed experiments, performed measurements, analyzed and evaluated the data and prepared the manuscript. JXU designed experiments, prepared the QDs for immuno-staining. TD conceived of SOFI, analyzed and evaluated the data. AA conceived of the study. YS-T conceived of the study, participated in the design and helped to draft the manuscript. SW participated in the design and coordination of the study, evaluated the data and prepared the manuscript. All authors read and approved the final manuscript.

\section{Acknowledgements}

This work was supported by NIH grant \#5R01EB000312, NIH grant \#1R01GM086197 and the Marco Polo Program of University of Bologna. Work in the lab of YST is supported by the European Research Council (ERC).

\section{Author details}

${ }^{1}$ Department of Chemistry and Biochemistry, Department of Physiology, and the California NanoSystem Institute (CNSI), University of California Los Angeles, Los Angeles, CA 90095-1569, USA. ²Department of Chemistry "G. Ciamician", University of Bologna, Via Selmi 2, Bologna 40126, Italy. ${ }^{3}$ SOFast GmbH, Dresdener Str 14, Berlin 10999, Germany. ${ }^{4}$ The Mina \& Everard Goodman Faculty of Life Sciences \& Institute of Nanotechnology, Bar-Ilan University, Ramat Gan 52900, Israel.

Received: 20 August 2012 Accepted: 7 February 2013

Published: 25 February 2013

\section{References}

Aizer A, Shav-Tal Y (2008) Intracellular trafficking and dynamics of $P$ bodies. Prion 2(4):131-134. doi:10.4161/pri.2.4.7773

Aizer A, Brody Y, Ler LW, Sonenberg N, Singer RH, Shav-Tal Y (2008) The dynamics of mammalian $\mathrm{P}$ body transport, assembly, and disassembly in vivo. Mol Biol Cell 19(10):4154-4166. doi:10.1091/mbc.E08-05-0513

Bates M, Huang B, Dempsey GT, Zhuang X (2007) Multicolor Super-Resolution Imaging with Photo-Switchable Fluorescent Probes. Science (New York, NY) 317:1749-1753. doi:10.1126/science.1146598

Betzig E, Patterson GH, Sougrat R, Lindwasser OW, Olenych S, Bonifacino JS, Davidson MW, Lippincott-Schwartz J, Hess HF (2006) Imaging Intracellular Fluorescent Proteins at Nanometer Resolution. Science (New York, NY) 313:1642-1645. doi:10.1126/science.1127344

Burnette DT, Sengupta P, Dai Y, Lippincott-Schwartz J, Kachar B (2011) Bleaching/ blinking assisted localization microscopy for superresolution imaging using standard fluorescent molecules. PNAS 108(52):21081-21086. doi:10.1073/ pnas.1117430109

Carbonaro M, O'Brate A, Giannakakou P (2011) Microtubule disruption targets HIF-1alpha mRNA to cytoplasmic P-bodies for translational repression. J Cell Biol 192(1):83-99. doi:10.1083/jcb.201004145

De Heredia ML, Jansen R-P (2004) mRNA localization and the cytoskeleton. Curr Op Cell Biol 16(1):80-85. doi:10.1038/nbt895

Dedecker P, Mo GCH, Dertinger T, Zhang J (2012) Widely accessible method for superresolution fluorescence imaging of living systems. PNAS 109(27):10909-10914. doi:10.1073/pnas.1204917109

Dertinger T, Colyer R, lyer G, Weiss S, Enderlein J (2009) Fast, background-free, 3D super-resolution optical fluctuation imaging (SOFI). PNAS 106(29):22287-22292. doi:10.1073/pnas.0907866106

Dertinger T, Colyer R, Vogel R, Enderlein J, Weiss S (2010a) Achieving increased resolution and more pixels with Superresolution Optical Fluctuation Imaging (SOFI). Opt Express 18(18):18875-18885. doi:10.1364/OE.18.018875, OSA

Dertinger T, Heilemann M, Vogel R, Sauer M, Weiss S (2010b) Superresolution Optical Fluctuation Imaging with Organic Dyes. Angew Chem Int Ed 49 (49):9441-9443. doi:10.1002/anie.201004138

Dertinger T, Colyer R, Vogel R, Heilemann M, Sauer M, Enderlein J, Weiss S (2012a) Superresolution Optical Fluctuation Imaging (SOFI). Adv Exp Med Biol 733:17-21. doi:10.1007/978-94-007-2555-3_2

Dertinger T, Xu J, Foroutan Naini O, Vogel R, Weiss S (2012b) SOFI-based 3D superresolution sectioning with a widefield microscope. Opt Nanoscopy 1(2): doi:10.1186/2192-2853-1-2

Donnert G, Keller J, Wurm CA, Rizzoli SO, Westphal V, Schönle A, Jahn R, Jakobs S, Eggeling C et al (2007) Two-Color Far-Field Fluorescence Nanoscopy. Biophys J 92(8):L67-L69. doi:10.1529/biophysj.107.104497

Eulalio A, Behm-Ansmant I, Schweizer D, Izaurralde E (2007) P-Body Formation is a Consequence, not the cause, of RNA-Mediated Gene Silencing. Mol Cell Biol 27(11):3970-3981. doi:10.1128/MCB.00128-07

Geissbuehler S, Dellagiacoma C, Lasser T (2011) Comparison between SOFI and STORM. Biomed Opt Express 2(3):408-420. doi:10.1364/BOE.2.000408

Geissbuehler S, Bocchio NL, Dellagiacoma C, Berclaz C, Leutenegger M, Lasser T (2012) Mapping molecular statistics with balanced super-resolution optical fluctuation imaging (bSOFI). Opt Nanoscopy 1(4) doi:10.1186/2192-2853-1-4

Gustafsson MGL (2000) Surpassing the lateral resolution limit by a factor of two using structured illumination microscopy. J Microscopy 198(2):82-87. doi:10.1046/j.1365-2818.2000.00710

Heintzmann R, Jovin TM, Cremer C (2002) Saturated patterned excitation microscopy-a concept for optical resolution improvement. JOSA A 19 (8):1599-1609. doi:10.1364/JOSAA.19.001599, OSA

Hell SW (2003) Toward fluorescence nanoscopy. Nature Biotech 21:1347-1355. doi:10.1038/nbt895 
Hell SW, Wichmann J (1994) Breaking the diffraction resolution limit by stimulated emission: stimulated-emission-depletion fluorescence microscopy. Opt Let 19(11):780-782. doi:10.1364/OL.19.000780

Hess ST, Girirajan TPK, Mason MD (2006) Ultra-High Resolution Imaging by Fluorescence Photoactivation Localization Microscopy. Biophys J 91:4258-4272. doi:10.1529/biophysj.106.091116

Huang B, Wang W, Bates M, Zhuang X (2008) Three-Dimensional SuperResolution Imaging by Stochastic Optical Reconstruction Microscopy. Science (New York, NY) 319:810-813. doi:10.1126/science.1153529

James M (1988) Pattern Recognition. John Wiley and Sons, New York, pp 36-40

Kedersha N, Stoecklin G, Ayodele M, Yacono P, Lykke-Andersen J, Fritzler MJ, Scheuner D, Kaufman RJ, Golan DE et al (2005) Stress granules and processing bodies are dynamically linked sites of mRNP remodeling. J Cell Biol 169(6):871-884. doi:10.1083/jcb.200502088

Kuno M, Fromm DP, Hamann HF, Gallagher A, Nesbitt DJ (2001) "On"/"off" fluorescence intermittency of single semiconductor quantum dots. J Chem Phys 115(2):1028-1041. doi:10.1063/1.1377883

Lang M, Jegou T, Chung I, Richter K, Munch S, Udvarhelyi A, Cremer C, Hemmerich P, Engelhardt J (2010) Three-dimensional organization of promyelocytic leukemia nuclear bodies. J Cell Science 123(3):392-400 doi:10.1242/jcs.053496

Leung AKL, Calabrese JM, Sharp PA (2006) Quantitative analysis of Argonaute protein reveals microRNA-dependent localization to stress granules. PNAS 103(48):18125-18130. doi:10.1073/pnas.0608845103

Liu J, Valencia-Sanchez MA, Hannon GJ, Parker R (2005) MicroRNA-dependent localization of targeted mRNAs to mammalian P-bodies. Nat Cell Biol 7:719-723. doi:10.1038/ncb1274

Manders EMM, Verbeek FJ, Aten JA (1993) Measurement of co-localization of objects in dual-colour confocal images. J Microsc 169(3):375-382. doi:10.1111/j.1365-2818.1993.tb03313.x

Nakamura K, Watakabe A, Hioki H, Fujiyama F, Yasuyo T, Yamamori T, Kaneko T (2007) Transiently increased colocalization of vesicular glutamate transporters 1 and 2 at single axon terminals during postnatal development of mouse neocortex: a quantitative analysis with correlation coefficient. Eur J Neurosci 26(11):3054-3067. doi:10.1111/j.1460-9568.2007.05868.x

Pillai RS, Bhattacharyya SN, Artus CG, Zoller T, Cougot N, Basyuk E, Bertrand E, Filipowicz W (2005) Inhibition of translational initiation by Let-7 MicroRNA in human cells. Science 309(5740):1573-1576. doi:10.1126/science.1115079

Rodgers JL, Nicewander WA (1995) Thirteen ways to look at the correlation coefficient. Amer Stat 24:59-66

Rust MJ, Bates M, Zhuang X (2006) Sub-diffraction-limit imaging by stochastic optical reconstruction microscopy (STORM). Nat Meth 3:793-796. doi:10.1038/nmeth929

Sen GL, Blau HM (2005) Argonaute 2/RISC resides in sites of mammalian mRNA decay known as cytoplasmic bodies. Nat Cell Biol 7:633-636. doi:10.1038/ncb1265

Shav-Tal Y, Singer RH (2005) RNA localization. J Cell Science 118(18):4077-4081. doi:10.1242/jcs.02543

Shroff H, Galbraith CG, Galbraith JA, White H, Gillette J, Olenych S, Davidson MW, Betzig E (2007) Dual-color superresolution imaging of genetically expressed probes within individual adhesion complexes. PNAS 104(51):20308-20313. doi:10.1073/pnas.0710517105

Simonson PD, Rothenberg E, Selvin PR (2011) Single-Molecule-Based SuperResolution Images in the Presence of Multiple Fluorophores. Nano Lett 11 (11):5090-5096. doi:10.1021/nl203560r

Weckwerth W (2007) Metabolomics: methods and protocols. Humana Pr Inc., pp 3-10

Weil TT, Parton RM, Herpers B, Soetaert J, Veenendaal T, Xanthakis D, Dobbie IM, Halstead JM, Hayashi R, Rabouille C, Davis I (2012) Drosophila patterning is established by differential association of mRNAs with P bodies. Nat Cell Biol 14(12):1305-15. doi:10.1038/ncb2627

Yang Z (2004) GW182 is critical for the stability of GW bodies expressed during the cell cycle and cell proliferation. J Cell Science 117(23):5567-5578. doi:10.1242/jcs.01477

doi:10.1186/2192-2853-2-2

Cite this article as: Gallina et al:: Resolving the spatial relationship between intracellular components by dual color super resolution optical fluctuations imaging (SOFI). Optical Nanoscopy 2013 2:2.

\section{Submit your manuscript to a SpringerOpen ${ }^{\circ}$ journal and benefit from:}

- Convenient online submission

- Rigorous peer review

- Immediate publication on acceptance

- Open access: articles freely available online

- High visibility within the field

- Retaining the copyright to your article

Submit your next manuscript at $>$ springeropen.com 